\title{
Downlink Transmission of Broadband OFCDM Systems-Part II: Effect of Doppler Shift
}

\author{
Yiqing Zhou, Member, IEEE, Jiangzhou Wang, Senior Member, IEEE, and Mamoru Sawahashi, Member, IEEE
}

\begin{abstract}
The orthogonal frequency and code division multiplexing (OFCDM) system with 2-D spreading (time- and frequency-domain spreading) is becoming a promising candidate for future broadband wireless communication systems. OFCDM is more attractive than orthogonal frequency-division multiplexing (OFDM) both by introducing frequency-domain spreading for frequency diversity provision and time-domain spreading for flexible data rate provision. To provide high-speed mobile services, multicode transmission is employed in conjunction with OFCDM. In a Gaussian or flat-fading channel, multicode channels are orthogonal. However, in a realistic wireless channel, the orthogonality no longer maintains. Thus, multicode interference (MCI) is caused. This paper focuses on the investigation of the effect of Doppler shift on the downlink transmission of high-speed mobile OFCDM systems. A practical channel estimation algorithm based on a code-multiplexed pilot channel is employed to track the variations of fading channels. Hybrid MCI cancellation and minimum mean-square error (MMSE) detection proposed by the authors is employed as an efficient way to eliminate the MCI in the frequency domain. The system performance is analytically studied with imperfect channel estimation to show how it is affected by parameters such as the window size in the channel estimation, Doppler shift, the number of stages of the hybrid detection, the power ratio of pilot to data channels, spreading factor, and so on.
\end{abstract}

Index Terms-Channel estimation, Doppler shift, fading channels, interference cancellation, minimum mean-square error (MMSE) detection, multicarrier transmission, orthogonal frequency-division multiplexing (OFDM), 2-D spreading.

\section{INTRODUCTION}

$\mathbf{R}$ ECENTLY, the research on the future fourthth generation (4G) wireless communication networks has drawn a lot of attention. Using a huge bandwidth of $100 \mathrm{MHz}$, the $4 \mathrm{G}$ system intends to provide mobile data at a rate of 100 Mbps or more, especially in downlink. Various multiple access schemes have been investigated for the broadband downlink transmission. Since the multicarrier access scheme, or orthogonal frequency-division multiplexing (OFDM), is robust to the severe multipath interference (MPI) occurring in the broadband channel, it is preferred in the $4 \mathrm{G}$ downlink transmission to

Paper approved by S. Ulukus, the Editor for Modulation and Signal Design of the IEEE Communications Society. Manuscript received February 13, 2004; revised January 5, 2005 and September 20, 2005. This paper was presented in part at the IEEE International Conference on Communications, Paris, France, June 2004.

Y. Zhou is with the Department of Electrical and Electronic Engineering, University of Hong Kong, Hong Kong (e-mail: yqzhou @eee.hku.hk).

J. Wang is with the Department of Electronics, University of Kent, Canterbury, Kent, CT2 7NT, U.K. (e-mail: j.z.wang@kent.ac.uk).

M. Sawahashi is with the IP Radio Network Development Department, NTT DoCoMo, Inc., Kanagawa 239-8536, Japan (e-mail: sawahasi@ mlab.yrp. nttdocomo.co.jp)

Digital Object Identifier 10.1109/TCOMM.2006.876872

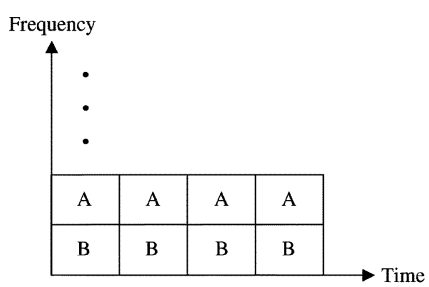

(a)

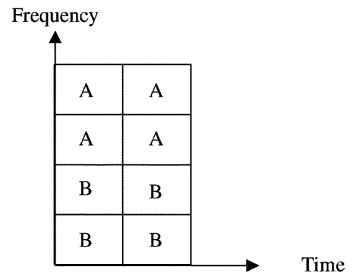

(c)

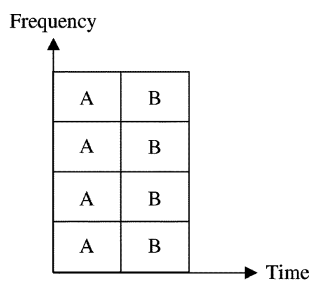

(b)

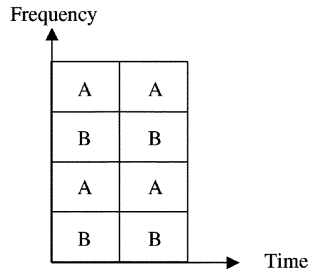

(d)
Fig. 1. Different spreading schemes in multicarrier systems (A and B are data symbols and OVSF code takes all " +1 "). (a) Spreading in time domain (MC-DS-CDMA) (4x1). (b) Spreading in frequency domain (MC-CDMA) $(1 \times 4)$. (c) 2-D spreading $(2 \times 2)$. (d) 2-D spreading with frequency-domain interleaving $(2 \times 2)$.

the conventional single carrier direct-sequence code-division multiple-access (DS-CDMA) scheme [1]. Spreading has been introduced in OFDM systems to provide flexibility in a multiuser environment. The OFDM with spreading in only time [2] and frequency domain [3] is known as multicarrier DS-CDMA (MC-DS-CDMA) and MC-CDMA, respectively. Moreover, the multicarrier system with 2-D spreading was independently proposed in [4] and [5], where spreading is employed in both time and frequency domains. Denoting the time-domain spreading factor as $N_{T}$ and frequency-domain spreading factor as $N_{F}$, the total spreading factor $N$ is $N=N_{T} \times N_{F}$. As an example, Figs. 1(a)-(c) illustrate time-domain, frequency-domain, and 2-D spreading schemes, respectively, in multicarrier systems, where two data symbols, A and B, are spread using the all " +1 " orthogonal variable spreading factor (OVSF) code [6] with a length of four. It can be seen that instead of placing the spread chips in only time or frequency domain, the 2-D spreading locates the chips in blocks on the time-frequency grid. Basically, frequency-domain spreading introduces frequency diversity, whereas time-domain spreading mainly provides flexibility of different data rates. Fig. 1(d) shows 2-D spreading with frequency interleaving [7], which is used to separate the subcarriers carrying the same information to get different fading for larger frequency diversity gain. It is expected that 2-D spreading with frequency interleaving is the most attractive among the four schemes. 
Based on 2-D spreading with interleaving, an orthogonal frequency and code division multiplexing (OFCDM) system was proposed for the downlink transmission in future $4 \mathrm{G}$ networks [7]-[11], where multiple code channels can be assigned to one single user to provide high-speed data transmission. In most cases, the system works in a fading channel with a small Doppler shift, where the channel variation in one packet duration is negligible. Time-domain spreading can maintain the orthogonality among the code channels. On the other hand, on interleaved subcarriers, the channel fading changes significantly due to the high frequency selectivity in a broadband channel. Thus, system performance is determined by the trade-off between the multicode interference (MCI) and the frequency diversity gain both resulting from the frequency selectivity. Under the conditions of light channel loads, quaternary phase-shift keying (QPSK) modulation, and small delay spread, the system performance can be improved by increasing the frequency-domain spreading factor. A simple equal gain combining (EGC) and the minimum mean-square error (MMSE) combining have been studied for time-domain despreading and frequency-domain despreading, respectively [7], [9].

A promising detection technique, called hybrid MCI cancellation and MMSE detection, has been proposed for the OFCDM system [12]. It has been shown in [12] that the hybrid detection can significantly improve the performance of the OFCDM system by cancelling out MCI in frequency domain (MCI-F) when Doppler shift is zero. In this paper, further investigation is carried out on the OFCDM system with the hybrid detection in the presence of Doppler shift. For a carrier frequency of $5 \mathrm{GHz}$, when a bullet train runs at the speed of $300 \mathrm{~km} / \mathrm{hr}$, the resultant maximum Doppler frequency $\left(f_{D}\right)$ can be as high as $1500 \mathrm{~Hz}$. In this case, even with a short packet length such as $0.5 \mathrm{~ms}$, the channel variation in one packet duration is not negligible. Thus, spreading in time domain cannot preserve the orthogonality between code channels and MCI in time domain (MCI-T) occurs. In the presence of Doppler shift, system performance will be quite different from that when the shift is zero. It is important to investigate the performance of the OFCDM system with the hybrid detection in a large range of Doppler shift.

This paper investigates the effect of Doppler shift on the hybrid detection for high-speed OFCDM systems. A flexible channel estimation algorithm based on the code-multiplexed pilot channel is presented, where the estimation window size can be chosen according to Doppler shift. Thus, the proposed algorithm can adapt to different channel conditions. Furthermore, analytical expressions are derived for the MMSE weights stage by stage and used for performance evaluation. The paper is organized as follows. The system model is described in Section II. Section III presents the analysis on the performance of the OFCDM system. Section V presents some representative numerical results. Finally, Section IV draws conclusions.

\section{SYSTEM DESCRIPTION}

\section{A. Transmitter Model}

In the OFCDM system with 2-D spreading, suppose that totally $K\left(K<N=N_{T} \times N_{F}\right)$ code-multi- plexed data channels be assigned to one user to provide high-speed data transmission. The $\bar{k}$ th data channel employs the 2-D spreading code $\left\{C_{N_{T}}^{\left(\bar{k}_{T}\right)}, C_{N_{F}}^{\left(\bar{k}_{F}\right)}\right\}$, where $\bar{k}=0,1, \cdots, K-1$ represents the code index. The time-domain spreading code $C_{N_{T}}^{\left(\bar{k}_{T}\right)}=\left\{c_{N_{T}, 0}^{\left(\bar{k}_{T}\right)}, \cdots, c_{N_{T}, N_{T}-1}^{\left(\bar{k}_{T}\right)}\right\}$ is an OVSF code with a length of $N_{T}$, which is the $\bar{k}_{T}$ th code at the $\left(\log _{2} N_{T}\right)$ th level in the code tree [6]. Similarly, the frequency-domain spreading code is denoted as $C_{N_{F}}^{\left(\bar{k}_{F}\right)}=\left\{c_{N_{F}, 0}^{\left(\bar{k}_{F}\right)}, \cdots, c_{N_{F}, N_{F}-1}^{\left(\bar{k}_{F}\right)}\right\}$. Excluding the 2-D code $\left\{C_{N_{T}}^{\left(\bar{k}_{T}\right)}, C_{N_{F}}^{\left(\bar{k}_{F}\right)}\right\}$, the rest $K-1$ codes can be divided into two subsets: one set, $\Omega_{T}=\left\{\left\{C_{N_{T}}^{\left(k_{T}\right)}, C_{N_{F}}^{\left(k_{F}\right)}\right\} \mid k_{T} \neq \bar{k}_{T}\right\}$, with different time-domain spreading codes from $C_{N_{T}}^{\left(\bar{k}_{T}\right)}$, and the other set, $\Omega_{F}=\left\{\left\{C_{N_{T}}^{\left(\bar{k}_{T}\right)}, C_{N_{F}}^{\left(k_{F}\right)}\right\} \mid k_{F} \neq \bar{k}_{F}\right\}$, with the same time-domain spreading code but different frequency-domain spreading codes from $C_{N_{F}}^{\left(\bar{k}_{F}\right)}$ [12]. The code channels in $\Omega_{T}$ must be orthogonal to the $\bar{k}$ th data channel in time domain, while the code channels in $\Omega_{F}$ are orthogonal to the $\bar{k}$ th data channel only in frequency domain. Generally, the MCI-F should be much more serious than MCI-T due to different fading on interleaved subcarriers. To keep the MCI small, the 2-D codes with the same frequency-domain spreading code, but different time-domain spreading codes should be assigned to data channels first. The number of interfering code channels in $\Omega_{F}$ is at most $I_{F}=\left\lfloor(K-1) /\left(N_{T}-1\right)\right\rfloor$, where $\lfloor x\rfloor$ is the integer portion of $x$, and the number of code channels in $\Omega_{T}$ is $I_{T}=K-1-I_{F}$. Moreover, the relationship between $\bar{k}, \bar{k}_{T}$ and $\bar{k}_{F}$ is given by $\bar{k}_{T}=\bar{k} \%\left(N_{T}-1\right)+1\left(\bar{k}_{T}=1, \cdots, N_{T}-1\right)$ and $\bar{k}_{F}=\left\lfloor\bar{k} /\left(N_{T}-1\right)\right\rfloor\left(\bar{k}_{F}=0,1, \cdots, N_{F}-1\right)$, where $\%$ stands for the modulo operation.

The downlink transmission of the broadband wireless communication systems is considered. Since the operations on all data symbols are similar, Fig. 2 shows the simplified block diagram of signal processing on one data symbol, when both scrambler/descrambler and waveform shaping filter are omitted. It can be seen that first the QPSK-modulated data symbol is two-dimensionally spread with $N_{T}$ chips in time domain and $N_{F}$ chips in frequency domain, then the signals from the pilot and all data code channels are added together by a code multiplexer. Note that for simple realization, the same known QPSK-modulated pilot symbol is used for the channel estimation for all subcarriers and time positions. The all " +1 " OVSF code is assigned to the pilot channel. Therefore, the time-domain spreading of the pilot symbol can be simply realized by a repeater. After multiplexing, the $N_{F}$ signals should be modulated to $N_{F}$ subcarriers. In the broadband OFCDM system, there are totally $M$ subcarriers and $N_{F} \ll M$. With $M$ subcarriers and frequency-domain spreading factor $N_{F}, N_{B}=M / N_{F}$ data symbols can be transmitted in parallel at the same time on each data code channel. Using a frequency-domain interleaver, the $N_{F}$ subcarriers carrying the same data symbol are separated as far as possible, so that the system can benefit from frequency diversity due to different fading on interleaved subcarriers. Although MCI-F will be increased by the frequency interleaver, it can be effectively cancelled out by MCI cancellation. The multi-carrier modulator can be realized by inverse fast Fourier transform (IFFT) in practical systems. 


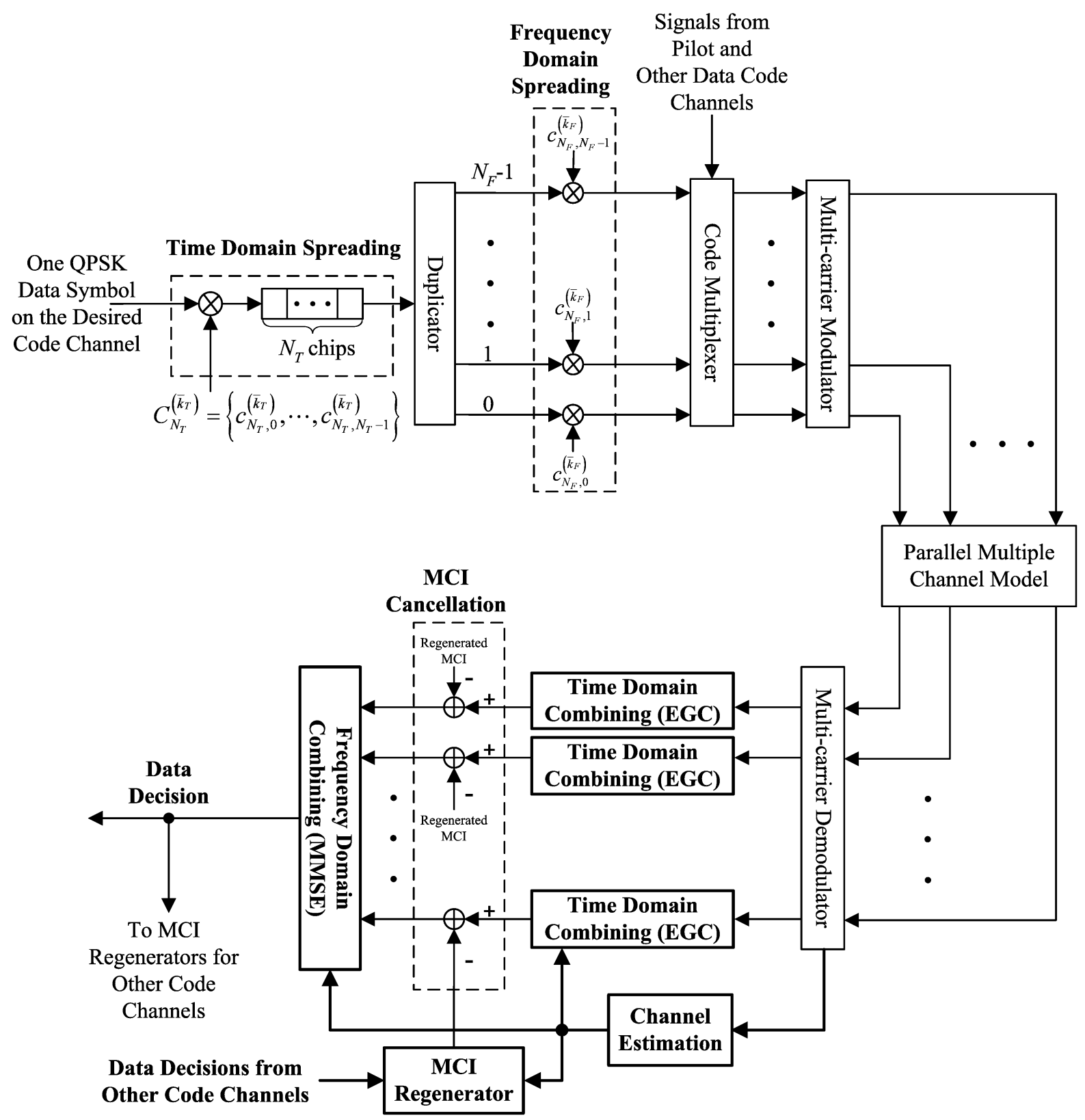

Fig. 2. Block diagram for signal processing of one data symbol.

In summary, the baseband transmitted data signal of the $m$ th subcarrier on the $i$ th OFCDM symbol in time domain can be expressed as

$$
S_{m, i}(t)=\sqrt{P} \sum_{k=0}^{K-1} d_{k, m, i} c_{N_{T}, i}^{\left(k_{T}\right)} c_{N_{F}, m}^{\left(k_{F}\right)} e^{j 2 \pi f_{m}(t-i T)} p(t-i T)
$$

where $P$ is the signal power of one data symbol, $d_{k, m, i}$ is the data symbol of the $k$ th code channel with $\left|d_{k, m, i}\right|^{2}=1$, $c_{N_{F}, m}^{\left(k_{F}\right)} c_{N_{T}, i}^{\left(k_{T}\right)}$ is the 2-D spreading code, $p(t)$ is the impulse response (unitary rectangular) of the pulse-shaping filter, $f_{m}=m / T_{e}$ represents the baseband-equivalent frequency of the $m$ th subcarrier, $T_{e}$ stands for the effective duration of a time chip (or OFCDM symbol), and $T=T_{e}+T_{g}$ is the complete duration including the guard interval $T_{g}$.
Since the OVSF code with all +1 is assigned to the pilot channel, the baseband pilot signal of the $m$ th subcarrier on the $i$ th OFCDM symbol can be expressed as

$$
S_{P, m, i}(t)=\sqrt{\beta P} d_{P} e^{j 2 \pi f_{m}(t-i T)} p(t-i T)
$$

where $\beta$ is the power ratio of pilot to one data channel and $d_{P}$ is the known pilot symbol with $\left|d_{P}\right|^{2}=1$. Suppose that there are totally $N_{d}$ OFCDM symbols in one packet duration. The baseband-equivalent complex transmitted signal in one packet duration is then given by

$$
S(t)=\sum_{m=0}^{M-1} \sum_{i=0}^{N_{d}-1}\left[S_{m, i}(t)+S_{P, m, i}(t)\right]
$$




\section{B. Channel and Receiver Models}

Assuming perfect suppression of multipath by the guard interval $T_{g}$, a parallel flat fading multichannel model is employed [13]. $H_{m}(t)$ is used to denote the complex channel fading for the $m$ th subcarrier. The amplitude and phase of $H_{m}(t)$ are Rayleigh distributed with $E\left\{\left|H_{m}(t)\right|^{2}\right\}=1$ and uniformly distributed in $[0,2 \pi)$, respectively. Moreover, the correlation coefficient of $H_{m}(t)$ based on time and frequency separation is given by [14]

$$
\begin{aligned}
\rho\left(m_{0}, m_{1}, t_{0}, t_{1}\right) & =\frac{E\left\{H_{m_{0}}\left(t_{0}\right) H_{m_{1}}^{*}\left(t_{1}\right)\right\}}{E\left\{\left|H_{m}(t)\right|^{2}\right\}} \\
& =\frac{J_{0}\left(2 \pi f_{D}\left|t_{0}-t_{1}\right|\right)}{\sqrt{1+\left(\left|f_{m_{0}}-f_{m_{1}}\right| / \Delta f_{c}\right)^{2}}}
\end{aligned}
$$

where $(\cdot)^{*}$ stands for the conjugate operation, $\left|t_{0}-t_{1}\right|$ is the time difference, $\left|f_{m_{0}}-f_{m_{1}}\right|$ is the frequency separation, $\Delta f_{c}$ is the channel coherence bandwidth, and $J_{0}(\cdot)$ is the correlation coefficient (zeroth-order Bessel function of the first kind).

After passing through the parallel multichannel, as shown in Fig. 2, the received equivalent baseband signals are first multicarrier demodulated, which can be realized by fast Fourier transform (FFT). Then the $N_{F}$ chips involved with $N_{F}$ subcarriers are obtained. On one hand, the output passes through the channel estimator. On the other hand, using the phase information of the estimated channels, the output is weighted by EGC and accumulated by the time-domain despreader for data channels. The resultant data signals will be further processed in terms of the hybrid detection. When the MCI canceller is employed, the output from the time-domain despreader for data channels is subtracted by a regenerated MCI. The remainders are then multiplied by weights obtained from MMSE algorithms and combined at the frequency-domain despreader to get the signals of the desired code channel. After frequency-domain despreading, a hard decision will be made to recover QPSK symbols. Finally, information bits at the desired code channel are obtained. The recovered information bits from all other code channels will be used to regenerate the MCI-F for the desired code channel. Basically, the interference regenerator performs the operation like the transmitter except the channel information. Although MCI cancellation cannot be performed at the zeroth stage due to unavailable information data, starting from the 1st stage, the cancellation process will continue in an iterative way, until a specified number of stages is reached.

\section{PERformanCE ANALYSIS}

After passing through the broadband channel and corrupted by a complex additive white Gaussian noise (AWGN) $\eta(t)$, the received baseband signal is denoted as $r(t)$. By setting $T_{g}$ larger than the maximum channel delay difference, there is no interference from adjacent OFCDM symbols. Thus, the output of the matched filter on the $\bar{m}$ th subcarrier in the $\bar{i}$ th OFCDM symbol duration can be expressed as

$$
\begin{aligned}
r_{\bar{m}, \bar{i}}= & \frac{1}{T_{e}} \int_{\bar{i} T}^{\bar{i} T+T_{e}} r(t) e^{-j 2 \pi f_{\bar{m}}(t-\bar{i} T)} p\left(T_{e}-(t-\bar{i} T) d t\right. \\
= & \frac{1}{T_{e}} \int_{\bar{i} T}^{\bar{i} T+T_{e}}\left[\sum_{m=0}^{M-1} \sum_{i=0}^{N_{d}-1}\left[S_{m, i}(t)+S_{P, m, i}(t)\right] H_{m}(t)\right. \\
\approx & \sqrt{P} \sum_{k=0}^{K-1} d_{k, \bar{m}, \bar{i}} c_{N_{T}, \bar{i}}^{\left(k_{T}\right)} c_{N_{F}, \bar{m}}^{\left(k_{F}\right)} \lambda_{\bar{m}, \bar{i}} \\
& +\sqrt{\beta P} d_{P} \lambda_{\bar{m}, \bar{i}}+\eta_{\bar{m}, \bar{i}}
\end{aligned}
$$

where

$$
\lambda_{\bar{m}, \bar{i}}=\frac{1}{T_{e}} \int_{0}^{T_{e}} H_{\bar{m}}(t+\bar{i} T) d t
$$

is the channel fading on the $\bar{m}$ th subcarrier, $\eta_{\bar{m}, \bar{i}}$ is the noise component with zero mean and a variance of $\sigma_{n}^{2}=N_{0} / T_{e}$, and $N_{0}$ is the power spectral density of $\eta(t)$. Note that with a broad bandwidth around $100 \mathrm{MHz}$, although the total number of subcarriers can be as large as 1024, the frequency spacing between adjacent subcarriers is nearly $100 \mathrm{kHz}$ and still big. In this case, the effect of the intercarrier interference (ICI) caused by Doppler shift is insignificant as long as $f_{D}$ is less than $10 \mathrm{kHz}$ [11]. Therefore, the ICI term is ignored in $r_{\bar{m}, \bar{i}}$.

\section{A. Channel Estimation}

Considering packet transmission, channel estimation in this paper is more practical than that in [12]. Channel estimation is carried out on each subcarrier by using the code-multiplexed pilot channel. The pilot signal is first recovered by summing the

$$
\begin{aligned}
& \xi_{\bar{m}, n}=\frac{\frac{1}{N_{T, P}} \sum_{\bar{i}=n N_{T, P}}^{(n+1) N_{T, P}-1} r_{\bar{m}, \bar{i}}}{\sqrt{\beta P} d_{P}}=\underbrace{\frac{1}{N_{T, P}} \sum_{\bar{i}=n N_{T, P}}^{(n+1) N_{T, P}-1} \lambda_{\bar{m}, \bar{i}}}_{h_{\bar{m}, n}^{(P)}}+\underbrace{\frac{1}{N_{T, P} \sqrt{\beta P} d_{P}} \sum_{i=n N_{T, P}}^{(n+1) N_{T, P}-1} \eta_{\bar{m}, \bar{i}}}_{\eta_{\bar{m}, n}^{(P)}} \\
& +\underbrace{\frac{1}{N_{T, P} \sqrt{\beta P} d_{P}} \sum_{\bar{i}=n N_{T, P}}^{(n+1) N_{T, P}-1}\left(\sum_{k=0}^{K-1} \sqrt{P} d_{k, \bar{m}, \bar{i}} c_{N_{T}, \bar{i}}^{\left(k_{T}\right)} c_{N_{F}, \bar{m}}^{\left(k_{F}\right)}\right) \lambda_{\bar{m}, \bar{i}}}_{I_{T, \bar{m}, n}^{(P)}}
\end{aligned}
$$


received signal over a time-domain window from $i=n N_{T, P}$ to $i=(n+1) N_{T, P}-1$, then preliminary channel estimations can be obtained by dividing the pilot signal with the known pilot symbol, given in (7) at the bottom of the previous page, where $N_{T, P}$ is the window size of channel estimation in time domain and it must be integer times of $N_{T}, n=0,1, \cdots,\left(N_{d} / N_{T, P}\right)-$ 1 stands for the number of preliminary channel estimations in one packet duration, $h_{m, n}^{(P)}$ stands for useful channel information, $\eta_{m, n}^{(P)}$ is the background noise with a variance of $\sigma_{N o i, P}^{2}=$ $\sigma_{n}^{2} /\left(\beta P N_{T, P}\right)$, and $I_{T, \bar{m}, n}^{(P)}$ stands for the MCI-T caused by all data channels with a variance given by

$$
\begin{aligned}
\sigma_{I_{T}^{(P)}}^{2}= & E\left\{\left|I_{T, \bar{m}, n}^{(P)}\right|^{2}\right\}=\frac{1}{N_{T, P} N_{T} \beta} \\
\times & {\left[\sum_{\bar{i}_{0}=0}^{N_{T}-1} \sum_{\bar{i}_{1}=0}^{N_{T}-1} R_{H}\left(\bar{m}, \bar{m}, \bar{i}_{0}, \bar{i}_{1}\right)\right.} \\
& \left.\times\left(\sum_{k=0}^{K-1} c_{N_{T}, \bar{i}_{0}}^{\left(k_{T}\right)} c_{N_{T}, \bar{i}_{1}}^{\left(k_{T}\right)}\right)\right]
\end{aligned}
$$

where $R_{H}\left(m_{0}, m_{1}, i_{0}, i_{1}\right)$ is the autocorrelation of $\lambda_{m, i}$, given by

$$
\begin{aligned}
& R_{H}\left(m_{0}, m_{1}, i_{0}, i_{1}\right) \\
& \quad=E\left\{\lambda_{m_{0}, i_{0}} \lambda_{m_{1}, i_{1}}^{*}\right\} \\
& \quad=\frac{1}{T_{e}^{2}} \int_{-T_{e}}^{T_{e}} \rho\left(m_{0}, m_{1}, t+i_{0} T, i_{1} T\right)\left(T_{e}-|t|\right) d t .
\end{aligned}
$$

Note that the window size $N_{T, P}$ of the channel estimation can be taken as various values from $N_{T}$ to $N_{d}$, as long as both $N_{T, P} / N_{T}$ and $N_{d} / N_{T, P}$ are integers. For example, when $N_{T}=$ 8 and there are $N_{d}=48$ OFCDM symbols in one packet, $N_{T, P}$ can be $8,16,24$ and 48 .

Therefore, the channel estimation can be carried out as follows. First, in the preliminary channel estimation [see (7)], $N_{T, P}$ is set to a specific value according to the Doppler shift. When the shift appears to be small, $N_{T, P}$ can be as large as $N_{d}$ to reduce the background noise. On the other hand, if the shift is large, $N_{T, P}$ must be set to a value as small as $N_{T}$ to reduce the distortion introduced by the average operation in $h_{\bar{m}, n}^{(P)}$.
Secondly, using the $N_{d} / N_{T, P}$ preliminary channel estimations $\xi_{\bar{m}, n}$, the channel estimation on the $\bar{m}$ th subcarrier in the $i$ th OFCDM symbol duration, $\xi_{\bar{m}}(i)\left(i=0,1, \cdots, N_{d}-1\right)$, can be obtained by a weighted sum of $\xi_{\bar{m}, n}$, given by

$$
\xi_{\bar{m}}(i)=\sum_{n=0}^{\left(N_{d} / N_{T, P}\right)-1} \alpha_{n, i} \xi_{\bar{m}, n}
$$

Linear interpolation can be adopted to track the channel variations by assuming that $\xi_{\bar{m}, n}$ is located at the $\left[(n+0.5) N_{T, P}\right]$ th OFCDM symbol. Note that the averaging estimation over the whole packet can be taken as a special case of interpolation with $N_{T, P}=N_{d}$ and $\alpha_{n, i}=1$. To improve the reliability of channel estimation, assuming adjacent subcarrier channels are unchanged or little changed, $\xi_{\bar{m}}(i)$ can be further averaged over adjacent subcarriers in frequency domain for further noise reduction. Using a sliding window average, the final channel estimation $\widehat{\lambda}_{m, i}$ for the $m$ th subcarrier in the $i$ th OFCDM symbol duration is given by

$$
\begin{aligned}
\widehat{\lambda}_{m, i}= & \frac{1}{2 N_{\mathrm{avg}}+1} \sum_{\bar{m}=m-N_{\mathrm{avg}}}^{m+N_{\mathrm{avg}}} \xi_{\bar{m}}(i) \\
= & \frac{1}{2 N_{\mathrm{avg}}+1} \sum_{\bar{m}=m-N_{\mathrm{avg}}}^{m+N_{\mathrm{avg}}} \sum_{n=0}^{\left(N_{d} / N_{T, P}\right)-1} \alpha_{n, i} \\
& \times\left(\frac{1}{N_{T, P}} \sum_{\bar{i}=n N_{T, P}}^{(n+1) N_{T, P}-1} \lambda_{\bar{m}, \bar{i}}+\eta_{\bar{m}, n}^{(P)}+I_{T, \bar{m}, n}^{(P)}\right)
\end{aligned}
$$

where $2 N_{\text {avg }}+1$ is the size of the sliding window in frequency domain.

Defining the channel estimation error as $e_{m, i}=\lambda_{m, i}-\widehat{\lambda}_{m, i}$, the autocorrelation function of $e_{m, i}$ in time domain is shown in (12) at the bottom of the page. Then, for the $m$ th subcarrier, the variance of the channel estimation error in one packet duration is given by $\bar{\sigma}_{\text {err }}^{2}=\left(1 / N_{d}\right) \sum_{i=0}^{N_{d}-1} R_{\text {err }}(i, i)$. It can be seen from (12) that the channel estimation error depends not only on the interference plus noise term, but also on the channel autocorrelation $R_{H}\left(m_{0}, m_{1}, i_{0}, i_{1}\right)$ and the weighting factors $\alpha_{n, i}$. Using (12), optimal parameters can be found for the channel estimation in various channel conditions.

$$
\begin{aligned}
R_{\mathrm{err}}\left(i_{0}, i_{1}\right)= & E\left\{e_{m, i_{0}} e_{m, i_{1}}^{*}\right\} \\
= & R_{H}\left(m, m, i_{0}, i_{1}\right)+\frac{1}{2 N_{\mathrm{avg}}+1} \sum_{n=0}^{\left(N_{d} / N_{T, P}\right)-1} \alpha_{n, i_{0}} \alpha_{n, i_{1}}^{*}\left(\sigma_{N o i, P}^{2}+\sigma_{I_{T}^{(P)}}^{2}\right)+\frac{1}{\left(2 N_{\mathrm{avg}}+1\right)^{2}\left(N_{T, P}\right)^{2}} \\
& \times \sum_{n_{0}=0}^{\left.N_{d} / N_{T, P}\right)-1} \sum_{n_{1}=0}^{\left.m+N_{d} / N_{T, P}\right)-1} \alpha_{n_{0}, i_{0}} \alpha_{n_{1}, i_{1}}^{*} \sum_{\bar{m}_{0}=m-N_{\text {avg }}}^{m+N_{\text {avg }}} \sum_{\bar{m}_{1}=m-N_{\text {avg }}}^{m+N_{\text {avg }}} \sum_{\bar{i}_{0}=n_{0} N_{T, P}}^{\left(n_{0}+1\right) N_{T, P}-1} \sum_{\bar{i}_{1}=n_{1} N_{T, P}}^{\left(n_{1}+1\right) N_{T, P}-1} R_{H}\left(\bar{m}_{0}, \bar{m}_{1}, \bar{i}_{0}, \bar{i}_{1}\right) \\
& -\frac{1}{\left(2 N_{\mathrm{avg}}+1\right) N_{T, P}} \sum_{\bar{m}=m-N_{\mathrm{avg}}}^{m+N_{\mathrm{avg}}} \sum_{n=0}^{\left(N_{d} / N_{T, P}\right)-1} \sum_{\bar{i}=n N_{T, P}}^{(n+1) N_{T, P}-1}\left[\alpha_{n, i_{1}}^{*} R_{H}\left(m, \bar{m}, i_{0}, \bar{i}\right)+\alpha_{n, i_{0}} R_{H}\left(\bar{m}, m, \bar{i}, i_{1}\right)\right] \quad(12)
\end{aligned}
$$




\section{B. Time-Domain Despreading}

In one packet duration with $N_{d}$ OFCDM symbols and using $M$ subcarriers, the $\bar{k}$ th code channel can transmit $\left(N_{d} / N_{T}\right)$. $\left(M / N_{F}\right)$ data symbols. Consider one data symbol, $d_{\bar{k}}$, which is spread over the first $N_{T}$ OFCDM symbols in the time domain, i.e., $\bar{i}=0, \cdots, N_{T}-1$, and the $N_{F}$ interleaved subcarriers in the frequency domain. A simple EGC is employed in time-domain despreading to collect useful signals from different OFCDM symbols. The signal at the output of the time-domain despreader for the $\bar{k}$ th code channel is given by

$$
\begin{aligned}
\gamma_{\bar{k}, m N_{B}}= & \frac{1}{N_{T}} \sum_{\bar{i}=0}^{N_{T}-1} r_{m N_{B}, \bar{i}} e^{-j \widehat{\varphi}_{m N_{B}, \bar{i}}} c_{N_{T}, \bar{i}}^{\left(\bar{k}_{T}\right)} \\
= & \underbrace{\sqrt{P} d_{\bar{k}} c_{N_{F}, m}^{\left(\bar{k}_{F}\right)} \alpha\left(m N_{B}\right)}_{S_{\bar{k}, m N_{B}}}+\mathrm{MCI}_{\bar{k}, m N_{B}}^{(F)} \\
& +\operatorname{MCI}_{\bar{k}, m N_{B}}^{(T)}+\eta_{\bar{k}, m N_{B}}
\end{aligned}
$$

where $m=0, \cdots, N_{F}-1$, the phase for EGC weight is given by

$$
e^{-j \widehat{\varphi}_{m N_{B}, \bar{i}}}=\frac{\widehat{\lambda}_{m N_{B}, \bar{i}}^{*}}{\left|\widehat{\lambda}_{m N_{B}, \bar{i}}\right|}
$$

and $\alpha\left(m N_{B}\right)$ is given by

$$
\alpha\left(m N_{B}\right)=\frac{1}{N_{T}} \sum_{\bar{i}=0}^{N_{T}-1} \lambda_{m N_{B}, \bar{i}} e^{-j \widehat{\varphi}_{m N_{B}, \bar{i}}}
$$

$S_{\bar{k}, m N_{B}}$ is the useful signal, $\mathrm{MCI}_{\bar{k}, m N_{B}}^{(F)}$ is the MCI-F from the $I_{F}$ codes in $\Omega_{F}$, given by

$$
\operatorname{MCI}_{k, m N_{B}}^{(F)}=\sqrt{P} \sum_{k \in \Omega_{F}} d_{k} c_{N_{F}, m}^{\left(k_{F}\right)} \alpha\left(m N_{B}\right)
$$

$\operatorname{MCI}_{k}^{(T)}, m N_{B}$ is the MCI-T from the $I_{T}$ codes in $\Omega_{T}$ and the pilot channel due to nonzero Doppler shift, and $\eta_{\bar{k}, m N_{B}}$ is the background noise.

\section{Frequency-Domain Despreading}

In frequency-domain despreading, the outputs of the time-domain despreader are weighted by different factors, multiplied by a frequency-domain spreading code and then summed over $N_{F}$ interleaved subcarriers. However, in the despreading process, serious MCI is caused by different fading on interleaved subcarriers, so MCI cancellation plus MMSE detection must be employed. At the zeroth stage of MCI cancellation, the regenerated MCI is unavailable due to detected data unavailable. Thus pure MMSE detection is used to combine the signals from different interleaved subcarriers. The weights of pure MMSE for the $\bar{k}$ th data channel are given by

$$
\omega_{\bar{k}}\left(m N_{B}\right)=\frac{E\left\{\sqrt{P} d_{\bar{k}} \gamma_{\bar{k}, m N_{B}}^{*} \mid \widehat{\alpha}\left(m N_{B}\right)\right\} c_{N_{F}, m}^{\left(\bar{k}_{F}\right)}}{E\left\{\left|\gamma_{\bar{k}, m N_{B}}\right|^{2} \mid \widehat{\alpha}\left(m N_{B}\right)\right\}}
$$

where $\widehat{\alpha}\left(m N_{B}\right)=\left(1 / N_{T}\right) \sum_{\bar{i}=0}^{N_{T}-1}\left|\widehat{\lambda}_{m N_{B}, \bar{i}}\right|$. It can be seen in (16) that in the evaluation of $\omega_{\bar{k}}\left(m N_{B}\right)$, the power of $\gamma_{\bar{k}, m N_{B}}$ must be estimated. Suppose that the useful signal, residual MCI-F, MCI-T, and noise are independent to each other. The power of $\gamma_{\bar{k}, m N_{B}}$ is given by

$$
\begin{aligned}
E\left\{\left|\gamma_{\bar{k}, m N_{B}}\right|^{2} \mid \widehat{\alpha}\left(m N_{B}\right)\right\} \\
=E\left\{\left|S_{\bar{k}, m N_{B}}\right|^{2} \mid \widehat{\alpha}\left(m N_{B}\right)\right\} \\
+E\left\{\left|\operatorname{MCI}_{\bar{k}, m N_{B}}^{(F)}\right|^{2} \mid \widehat{\alpha}\left(m N_{B}\right)\right\} \\
+E\left\{\left|\operatorname{MCI}_{\bar{k}, m N_{B}}\right|^{(t)} \mid \widehat{\alpha}\left(m N_{B}\right)\right\} \\
+E\left\{\left|\eta_{\bar{k}, m N_{B}}\right|^{2} \mid \widehat{\alpha}\left(m N_{B}\right)\right\}
\end{aligned}
$$

where

$$
\begin{aligned}
& E\left\{\left.\left|S_{\bar{k}, m N_{B}}\right|\right|^{2} \mid \widehat{\alpha}\left(m N_{B}\right)\right\} \\
& =P\left|\widehat{\alpha}\left(m N_{B}\right)\right|^{2} \\
& E\left\{\left|\mathrm{MCI}_{\bar{k}, m N_{B}}^{(F)}\right|^{2} \mid \widehat{\alpha}\left(m N_{B}\right)\right\} \\
& \quad=\sigma_{\mathrm{MCI}-\mathrm{F}}^{2}=P I_{F}\left|\widehat{\alpha}\left(m N_{B}\right)\right|^{2} \\
& E\left\{\left.\left|\mathrm{MCI}_{\bar{k}, m N_{B}}^{(t)}\right|\right|^{2} \mid \widehat{\alpha}\left(m N_{B}\right)\right\}=\sigma_{\mathrm{MCI}-\mathrm{T}, \bar{k}}^{2} \\
& =P \sum_{k \in \Omega_{T}}\left[\frac{1}{N_{T}} \sum_{\bar{i}=0}^{N_{T}-1}\left|\hat{\lambda}_{m N_{B}, \bar{i}}\right| c_{N_{T}, \bar{i}}^{\left(k_{T}\right)} c_{N_{T}, \bar{i}}^{\left(\bar{k}_{T}\right)}\right]^{2} \\
& \quad+\beta P\left[\frac{1}{N_{T}} \sum_{\widehat{i}=0}^{N_{T}-1}\left|\hat{\lambda}_{m N_{B}, \bar{i}}\right| c_{N_{T}, \bar{i}}^{\left(\bar{k}_{T}\right)}\right]^{2}
\end{aligned}
$$

and

$$
E\left\{\left|\eta_{\bar{k}, m N_{B}}\right|^{2} \mid \widehat{\alpha}\left(m N_{B}\right)\right\}=\sigma_{n}^{2} / N_{T} .
$$

Therefore, $\omega_{\bar{k}}\left(m N_{B}\right)$ can be estimated as

$$
\begin{aligned}
& \omega_{\bar{k}}\left(m N_{B}\right) \\
& =\frac{P \widehat{\alpha}\left(m N_{B}\right)}{P\left|\widehat{\alpha}\left(m N_{B}\right)\right|^{2}+P I_{F}\left|\widehat{\alpha}\left(m N_{B}\right)\right|^{2}+\sigma_{\mathrm{MCI}-\mathrm{T}, \bar{k}}^{2}+\sigma_{n}^{2} / N_{T}}
\end{aligned}
$$

Note that for zero Doppler shift, $\sigma_{\mathrm{MCI}-\mathrm{T}, \bar{k}}^{2}$ is zero and the MMSE weight $\omega_{\bar{k}}\left(m N_{B}\right)$ is actually the same for all data channels as in [12]. However, when non-zero Doppler shift exists, $\sigma_{\mathrm{MCI}-\mathrm{T}, \bar{k}}^{2}$ is different for different $\bar{k}$. Therefore, $\omega_{\bar{k}}\left(m N_{B}\right)$ varies with different data channels. By summing the signals of the $N_{F}$ interleaved subcarriers, the output of the frequency-domain despreader is given by

$$
\begin{aligned}
y_{\bar{k}} & =\sum_{m=0}^{N_{F}-1} \gamma_{\bar{k}, m N_{B}}\left[c_{N_{F}, m}^{\left(\bar{k}_{F}\right)} \omega_{\bar{k}}\left(m N_{B}\right)\right] \\
& =S_{\bar{k}}+\operatorname{MCI}_{\bar{k}}^{(F)}+\operatorname{MCI}_{\bar{k}}^{(t)}+\eta_{\bar{k}}
\end{aligned}
$$


where $S_{\bar{k}}$ is the desired data signal, $\mathrm{MCI}_{\bar{k}}^{(F)}$ and $\mathrm{MCI}_{\bar{k}}^{(T)}$ are the MCI-F and MCI-T, respectively, and $\eta_{\bar{k}}$ is the background noise component. Finally, hard decision will be made on the output, $y_{\bar{k}}$, and tentative decision $d_{\bar{k}}^{(0)}$ will be obtained, where the superscript " 0 " stands for the zeroth stage. For QPSK modulation, the probabilities of incorrect decisions of real and imaginary parts of $y_{\bar{k}}$ are the same. A semi-analytical method [12] is employed to evaluate the bit-error rate (BER) of $y_{\bar{k}}$. Note that $\operatorname{MCI}_{\bar{k}}^{(F)}$ and $\mathrm{MCI}_{\bar{k}}^{(T)}$ are composed of $I_{F}$ and $I_{T}$ interference channels, respectively, where $I_{T} \gg I_{F}$. In performance evaluation, $\operatorname{MCI}_{\bar{k}}^{(T)}$ is modeled as AWGN, while the values of $S_{\bar{k}}$ and $\operatorname{MCI}_{\bar{k}}^{(F)}$ are calculated conditioned on data symbols $\left\{d_{k}, k=\bar{k}\right.$ or $\left.k \in \Omega_{F}\right\}$ and channel information $\left\{\lambda_{m, i}\right\}$ and $\left\{\hat{\lambda}_{m, i}\right\}$. Conditioned on the values of $S_{\bar{k}}$ and $\mathrm{MCI}_{\bar{k}}^{(F)}$, the BER of $y_{\bar{k}}$ can be obtained with the variances of $\mathrm{MCI}_{\bar{k}}^{\left(\frac{1}{T}\right)}$ and $\eta_{\bar{k}}$. Then the conditional BER is averaged over all possible data symbols and channel information, which can be numerically evaluated by a Monte Carlo Approach [15].

In order to reduce the severe MCI caused by different fading on interleaved subcarriers, multistage MCI cancellation is employed in the frequency domain with MMSE detection. Generally speaking, with the tentative data decisions $d_{0}^{(s-1)}, \cdots, d_{k, k \neq \bar{k}}^{(s-1)}, \cdots, d_{K-1}^{(s-1)}$, of the $(s-1)$ th stage and channel estimation $\hat{\lambda}_{\bar{m}, \bar{i}}$, the MCI-F can be regenerated at the $s$ th stage for the $\bar{k}$ th data channel on the $\left(m N_{B}\right)$ th subcarrier, given by

$$
Q_{\bar{k}, m N_{B}}^{(F)}(s)=\sqrt{P} \sum_{k \in \Omega_{F}} d_{k}^{(s-1)} c_{N_{F}, m}^{\left(k_{F}\right)} \widehat{\alpha}\left(m N_{B}\right)
$$

The output of the MCI cancellation is then given by

$$
\begin{aligned}
\gamma_{\bar{k}, m N_{B}}^{(s)}= & \gamma_{\bar{k}, m N_{B}}-Q_{\bar{k}, m N_{B}}^{(F)}(s) \\
= & S_{\bar{k}, m N_{B}}+\operatorname{MCI}_{\bar{k}, m N_{B}}^{(F)}(s) \\
& +\operatorname{MCI}_{\bar{k}, m N_{B}}^{(T)}+\eta_{\bar{k}, m N_{B}}
\end{aligned}
$$

where $S_{\bar{k}, m N_{B}}, \operatorname{MCI}_{\bar{k}, m N_{B}}^{(T)}$, and $\eta_{\bar{k}, m N_{B}}$ are the useful signal, MCI-T, and background noise component, respectively, which are the same as the corresponding terms in (13). However, $\operatorname{MCI}_{\bar{k}, m N_{B}}^{(F)}(s)$ is the residual MCI-F, given by

$$
\begin{aligned}
\operatorname{MCI}_{\bar{k}, m N_{B}}^{(F)}(s)= & \operatorname{MCI}_{\bar{k}, m N_{B}}^{(F)}-Q_{\bar{k}, m N_{B}}^{(F)}(s) \\
= & \sqrt{P} \sum_{k \in \Omega_{F}} d_{k} c_{N_{F}, m}^{\left(k_{F}\right)} \alpha\left(m N_{B}\right) \\
& -\sqrt{P} \sum_{k \in \Omega_{F}} d_{k}^{(s-1)} c_{N_{F}, m}^{\left(k_{F}\right)} \widehat{\alpha}\left(m N_{B}\right)
\end{aligned}
$$

The new weights of MMSE with MCI cancellation at the $s$ th stage is then given by

$$
\begin{aligned}
\omega_{\bar{k}}^{(s)}\left(m N_{B}\right) & =\frac{E\left\{\sqrt{P} d_{\bar{k}}\left[\gamma_{\bar{k}, m N_{B}}^{(s)}\right]^{*} \mid \widehat{\alpha}\left(m N_{B}\right)\right\} c_{N_{F}, m}^{\left(\bar{k}_{F}\right)}}{E\left\{\left|\gamma_{\bar{k}, m N_{B}}^{(s)}\right|^{2} \mid \widehat{\alpha}\left(m N_{B}\right)\right\}} \\
& =\frac{P \widehat{\alpha}\left(m N_{B}\right)}{E\left\{\left|\gamma_{\bar{k}, m N_{B}}^{(s)}\right|^{2} \mid \widehat{\alpha}\left(m N_{B}\right)\right\}}
\end{aligned}
$$

As to the power of $\gamma_{\bar{k}, m N_{B}}^{(s)}$, the power of useful signal and the variance of MCI-T and noise are unchanged as that in (18), (20), and (21), respectively. However, with good channel estimation, the variance of MCI-F $E\left\{\left|\operatorname{MCI}_{\bar{k}, m N_{B}}^{(F)}(s)\right|^{2} \mid \widehat{\alpha}\left(m N_{B}\right)\right\}$ reduces with stages due to the MCI reduction. At the $s$ th stage $(s \geq$ $1)$, assuming that the data decision error $d_{k}-d_{k}^{(s-1)}$ and the error $\alpha\left(m N_{B}\right)-\widehat{\alpha}\left(m N_{B}\right)$ are independent with zero mean, the variance of residual MCI-F is given by

$$
\begin{aligned}
\sigma_{\mathrm{MCIF}}^{2}(s)= & P \cdot E\left\{\mid \sum_{k \in \Omega_{F}} d_{k} \alpha\left(m N_{B}\right)\right. \\
& \left.\quad-\left.\sum_{k \in \Omega_{F}} d_{k}^{(s-1)} \widehat{\alpha}\left(m N_{B}\right)\right|^{2}\right\} \\
\approx & P \sum_{k \in \Omega_{F}}\left[E\left\{\left|\left(d_{k}-d_{k}^{(s-1)}\right) \cdot \widehat{\alpha}\left(m N_{B}\right)\right|^{2}\right\}\right. \\
= & 4 P P_{b}^{(s-1)} I_{F}\left|\widehat{\alpha}\left(m N_{B}\right)\right|^{2} \\
& +\frac{P I_{F}}{N_{T}^{2}} \sum_{i_{1}=0}^{N_{T}-1} \sum_{i_{2}=0}^{N_{T}-1} R_{\mathrm{err}}\left(i_{1}, i_{2}\right)
\end{aligned}
$$

where $P_{b}^{(s-1)}$ is the BER of the $(s-1)$ th stage. It can be seen from (28) that after MCI cancellation, on one hand, the variance of the original MCI-F is reduced from $P I_{F}\left|\alpha\left(m N_{B}\right)\right|^{2}$ (see (19)) to $4 P P_{b}^{(s-1)} I_{F}\left|\widehat{\alpha}\left(m N_{B}\right)\right|^{2}$, on the other hand, an extra error is introduced as the second term on the right hand side of (28) due to the channel estimation error. Therefore, the channel estimation error not only affects the BER of the previous stage $P_{b}^{(s-1)}$, but also increases the residual MCI of the current stage. Therefore, precise channel estimation is critical for the hybrid detection.

Finally, the output signal after hybrid detection is expressed as

$$
\begin{aligned}
y_{\bar{k}}^{(s)} & =\sum_{m=0}^{N_{F}-1} r_{\bar{k}, m N_{B}}^{(s)}\left[c_{N_{F}, m}^{\left(\bar{k}_{F}\right)} \omega_{\bar{k}}^{(s)}\left(m N_{B}\right)\right] \\
& =S_{\bar{k}}^{(s)}+\operatorname{MCI}_{\bar{k}}^{(F)}(s)+\operatorname{MCI}_{\bar{k}}^{(t)}(s)+\eta_{\bar{k}}^{(s)}
\end{aligned}
$$

where $S_{\bar{k}}^{(s)}$ is the desired signal component, $\operatorname{MCI}_{\bar{k}}^{(F)}(s)$ is the residual MCI-F, $\operatorname{MCI}_{\bar{k}}^{(T)}(s)$ is the MCI-T, and $\eta_{\bar{k}}^{(s)}$ is the noise at the $s$ th stage. Note that in the hybrid detection, not only the 


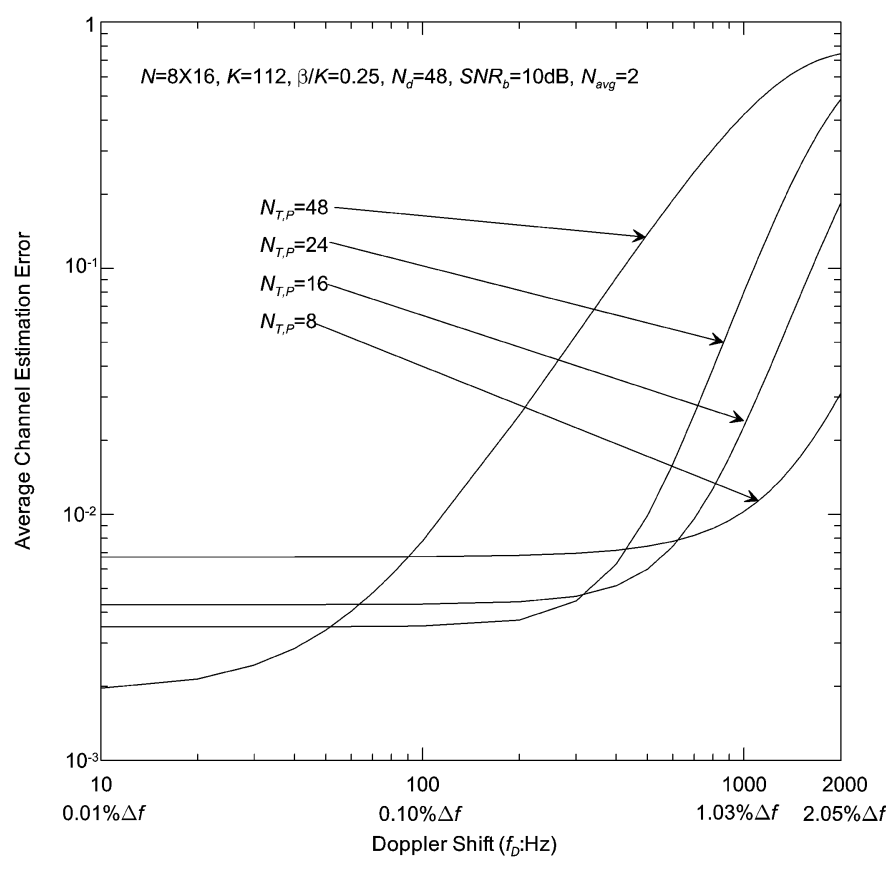

Fig. 3. Channel estimation error.

MCIs but also the useful signal and the background noise change with stages because of updating MMSE weights. The BER evaluation of $y_{\bar{k}}^{(s)}$ can be obtained using the semianalytical method described in Section IV of [12].

\section{NUMERICAL RESULtS}

Some representative numerical results are presented in this section. A broadband system with $100 \mathrm{MHz}$ is employed. Totally, $M=1024$ subcarriers are used and the resultant subcarrier spacing is about $\Delta f=97.7 \mathrm{kHz}$. The effective OFCDM symbol duration is $10.24 \mu \mathrm{s}$. According to the typical urban channel model [16], the delay spread of the multipath channel is around $2.14 \mu \mathrm{s}$. Hence, a guard interval $T_{g}$ of $2.26 \mu \mathrm{s}$ is adequate to combat the MPI. Therefore, the complete OFCDM symbol duration is $12.5 \mu \mathrm{s}$. There are totally $N_{d}=48$ OFCDM symbols in one packet duration. Unless noted otherwise, the average signal-to-noise ratio (SNR) per bit is defined as $\mathrm{SNR}_{b}=$ $\left(P / 2 \sigma_{n}^{2}\right) N(1+\beta / K)=10 \mathrm{~dB}$. The power ratio between the pilot channel and all data channels is $\beta / K=0.25$. The 2-D spreading factor is set to $N=8 \times 16$ and the system load is defined as $K / N=0.875$. The correlation coefficient between two adjacent interleaved subcarriers of interest is set to $\rho\left(0, N_{B}, t, t\right)=\rho(0,64, t, t) \approx 0.16$, corresponding to a coherence bandwidth $\Delta f_{c}$ of $1 \mathrm{MHz}$. The correlated Rayleigh fading channels are generated according to [17]. Finally, in the channel estimation, given $\mathrm{SNR}_{b}=10 \mathrm{~dB}$ and $\Delta f_{c}=1 \mathrm{MHz}$, the sliding window size in frequency domain $2 N_{\text {avg }}+1$ is set to 5 , which is the optimum size for $\Delta f_{c}$ of $1 \mathrm{MHz}$.

For a large Doppler shift, channel estimation plays an important role in the OFCDM system with hybrid detection. The performance of the channel estimation algorithm is first investigated and the results are shown in Fig. 3 for different window sizes $N_{T, P}$ of channel estimation. The Doppler shift ranges from 0 to $2000 \mathrm{~Hz}(0 \%$ to $2.05 \% \Delta f)$. Although it

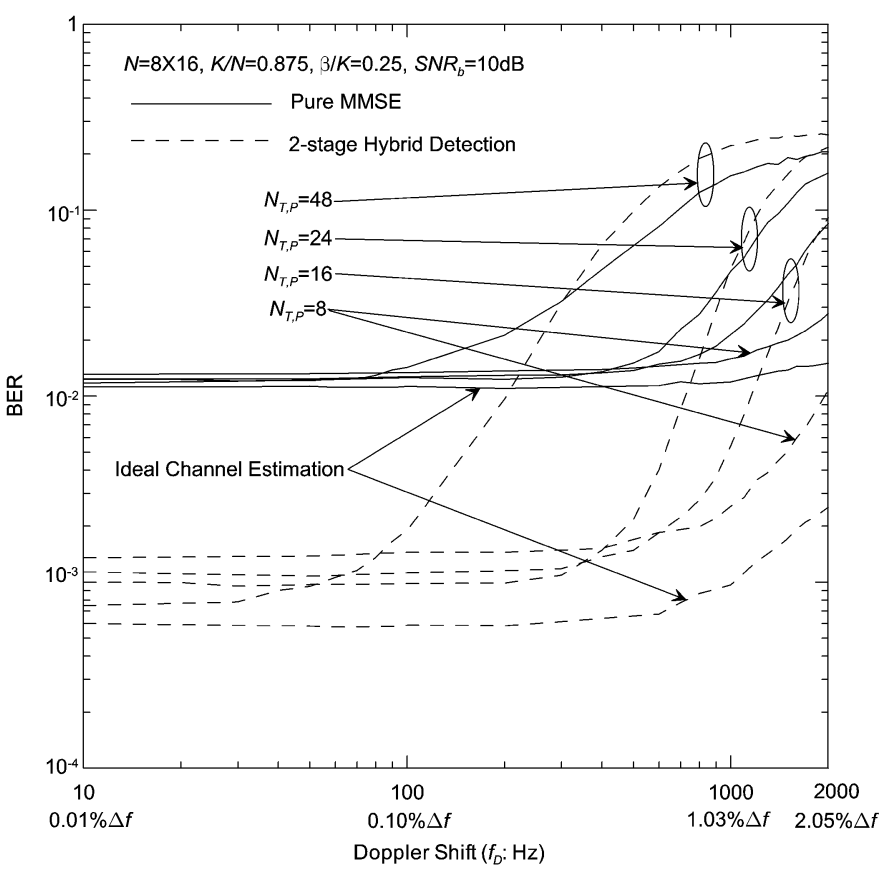

Fig. 4. System performance as a function of Doppler shift.

is not shown in the figure, the channel estimation error at $0 \leq f_{D}<10 \mathrm{~Hz}(0.01 \% \Delta f)$ is almost the same as that at $f_{D}=10 \mathrm{~Hz}$ for various $N_{T, P}$. To make the variance of channel estimation not be a function of time position, the variance is averaged over the $N_{d}$ time positions, denoted as the average channel estimation error. It can be seen that given $N_{T}=8$, the channel estimation with $N_{T, P}=N_{d}=48$ yields the smallest average channel estimation error for all possible values of $N_{T, P}$, when Doppler shift is very small, i.e., $0<f_{D} \leq 30 \mathrm{~Hz}(0.03 \% \Delta f)$. However, when the Doppler shift increases from $30 \mathrm{~Hz}$, the estimation error for $N_{T, P}=48$ increases rapidly, and surpasses that for all $N_{T, P}=24,16,8$ when $f_{D}>100 \mathrm{~Hz}(0.10 \% \Delta f)$. Although the estimation error keeps increasing for $N_{T, P}=48$, the errors for all other $N_{T, P}$ keeps unchanged as long as $f_{D}<300 \mathrm{~Hz}(0.31 \% \Delta f)$. When $f_{D}>600 \mathrm{~Hz}(0.62 \% \Delta f), N_{T, P}=8$ yields the smallest channel estimation error. This is because for a small Doppler shift, a larger $N_{T, P}$ helps reduce the background noise in the channel estimation and thus improves the estimation quality, while for a large Doppler shift, the distortion caused by large $N_{T, P}$ becomes serious and a shorter $N_{T, P}$ is preferred. In conclusion, when the Doppler shift is small, a large window size of channel estimation is needed, whereas when the Doppler shift is large, a small window size is preferable.

Fig. 4 illustrates the BER performance as a function of Doppler shifts when various window sizes of channel estimation, $N_{T, P}$, are considered. The BERs of pure MMSE (the zeroth stage) and two-stage hybrid detection are both shown. As a comparison, the system performance with perfect channel estimation is also shown. It can be seen that the trends in BER of the hybrid detection are similar to that in Fig. 3. The performance of the hybrid is very sensitive to Doppler shift. Good channel estimation quality is crucial to the hybrid detection. It can also be seen that the BER with perfect channel estimation 


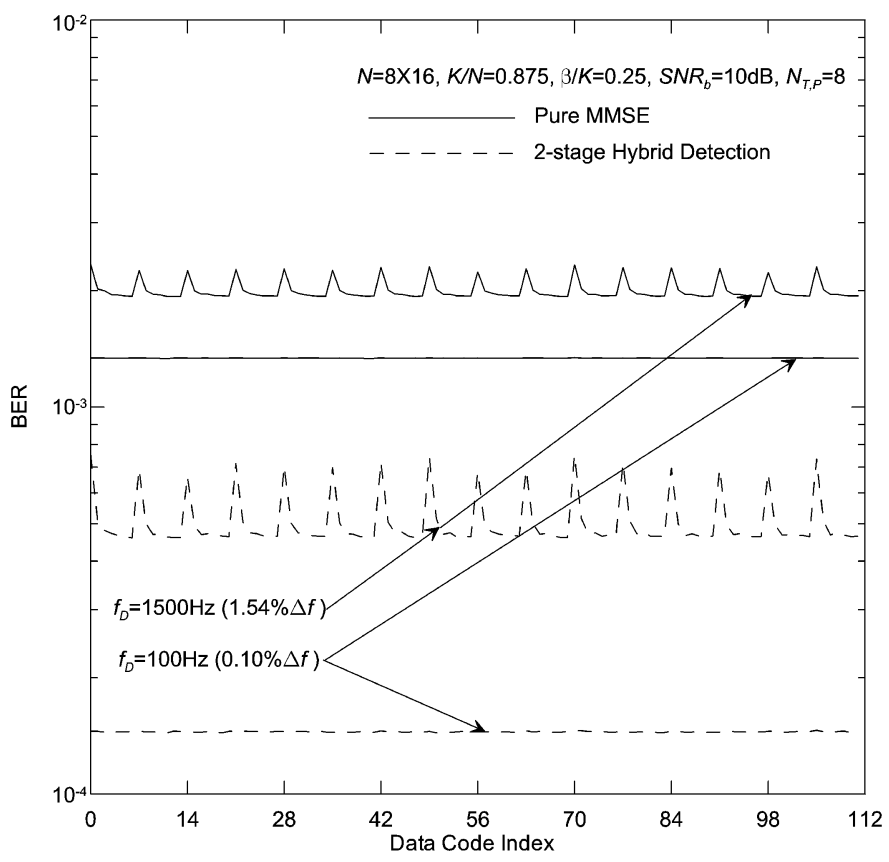

Fig. 5. BER performance versus different data code index.

increases with Doppler shift as well. This is because MCI-T becomes larger as $f_{D}$ increases, which cannot be cancelled out by the hybrid detection. Compared to the performance with perfect channel estimation, the BERs of $N_{T, P}=48,24,16$ degrade rapidly when the Doppler shift is larger than a certain value. On the other hand, the performance difference between $N_{T, P}=8$ and perfect channel estimation is almost unchanged, irrespective of Doppler. In the following, $N_{T, P}$ is set to $N_{T}$ to get stable performance for a large range of Doppler shift.

As described in the transmitter model, the pilot channel is code-multiplexed to data channels in time domain, and the spreading code with all " +1 " is assigned to the pilot channel. The spreading code of the pilot channel can be denoted as $C_{N_{T}}^{(0)}$. Given $N=8 \times 16$ and $K=112$, all the other time-domain spreading codes, $\left\{C_{N_{T}}^{\left(k_{T}\right)}, k_{T}=1, \cdots, N_{T}-1\right\}$ are assigned to the data channels with frequency-domain spreading codes, $\left\{C_{N_{F}}^{\left(k_{F}\right)}, k_{F}=0, \cdots, N_{F}-1\right\}$. Fig. 5 shows the BER performance on data code index. It can be seen that in for a small Doppler shift $f_{D}=100 \mathrm{~Hz}$, all data code channels have similar BER performance. However, for a large Doppler shift $f_{D}=1500 \mathrm{~Hz}(1.54 \% \Delta f)$, the BERs on different data code channels are different. The $(7 k)$ th $(k=0,1, \cdots, 15)$ data code channels have obviously higher BER than other data channels. This is due to that according to the code assignment scheme (as explained in the transmitter model), the $(7 k)$ th data code channels use $C_{N_{T}}^{(1)}$ as the time-domain spreading code. However, $C_{N_{T}}^{(1)}$ is adjacent to the pilot code, $C_{N_{T}}^{(0)}$, and they have the same mother code at the next higher layer [6]. Note that the pilot channel is highly powered with $\beta P=25 \% K P=28 P$. Therefore, for a large Doppler shift, the pilot channel causes serious MCI-T to the data channels using $C_{N_{T}}^{(1)}$, and the performance of these data code channels are obviously worse than other data channels. Therefore, if the data transmitted on different code channels have different levels of importance, the

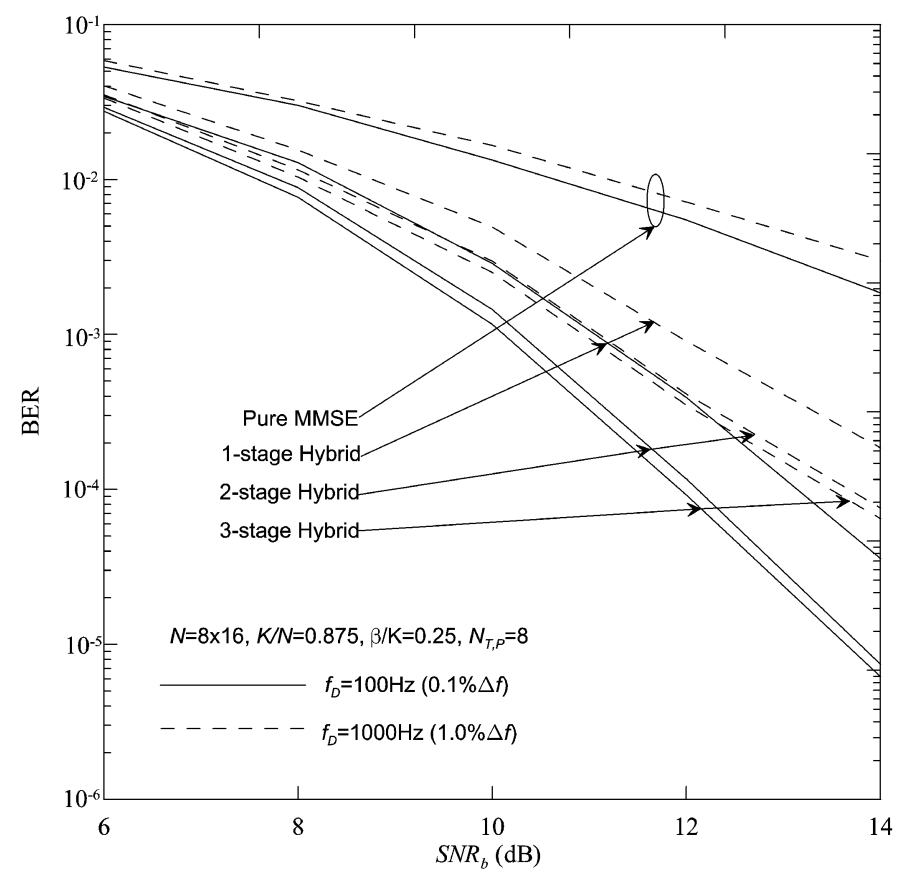

Fig. 6. Performance of hybrid detection as a function of $\mathrm{SNR}_{b}$.

code channels with $C_{N_{T}}^{(1)}$ should be used to transmit the least important information. On the other hand, in order to obtain same performance on all data channels, the channels using $C_{N_{T}}^{(1)}$ need higher level protection, which can be obtained by using lower-rate channel coding for example. Furthermore, when the system is not full-loaded, it should avoid assigning the time-domain spreading code $C_{N_{T}}^{(1)}$ to data channels.

The system performance is plotted in Fig. 6 as a function of $\mathrm{SNR}_{b}$. For both $f_{D}=100 \mathrm{~Hz}$ and $f_{D}=1000 \mathrm{~Hz}$, it is shown that the BER decreases as the number of stages increases due to the reduction of MCI in each stage, especially when SNR is large. The most significant reduction in BER is obtained from the zeroth stage to the first stage, then the improvement becomes smaller as the number of stages increases. It can also be seen that hybrid detection works even more effectively for $f_{D}=100 \mathrm{~Hz}$ than for $f_{D}=1000 \mathrm{~Hz}$. This is because for $f_{D}=100 \mathrm{~Hz}$, better channel estimation quality can be achieved than for $f_{D}=1000 \mathrm{~Hz}$. With better channel estimation, significant improvement can be obtained when $f_{D}=100 \mathrm{~Hz}$. When $f_{D}=1000 \mathrm{~Hz}$, the BER performance is worse than that for $f_{D}=100 \mathrm{~Hz}$ due to the channel estimation error and MCI-T which cannot be cancelled out by the hybrid detection. Although the BER performance gets better when the number of stages of MCI cancellation increases, it should be noted that the improvement becomes insignificant after the second stage for both $f_{D}=100 \mathrm{~Hz}$ and $f_{D}=1000 \mathrm{~Hz}$. Therefore, a two-stage hybrid detection is sufficient for QPSK at various values of SNR for a large range of Doppler shift.

Fig. 7 illustrates the effect of power ratio $\beta$ on the system performance for various Doppler shifts. It can be seen that with two-stage hybrid detection, when $\beta$ is small, the system performance degrades by the poor channel estimation. When $\beta$ increases, the BER performance improves as the quality of channel estimation improves, and BER reaches a minimum for 


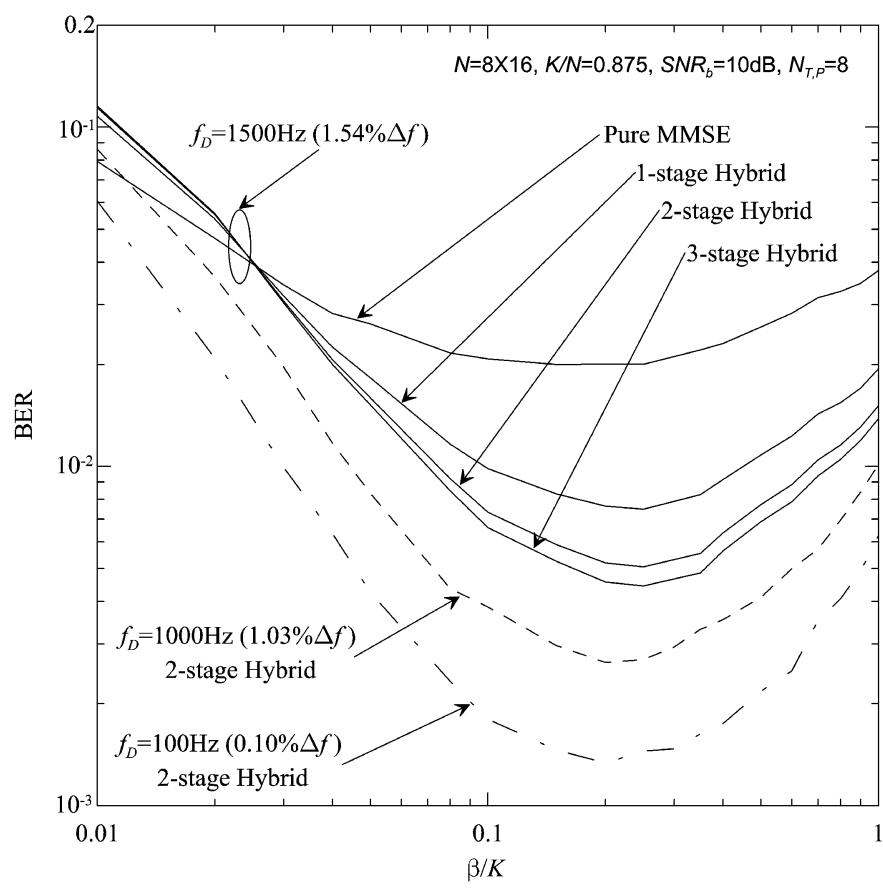

Fig. 7. System performance as a function of power ratio $\beta / K$.

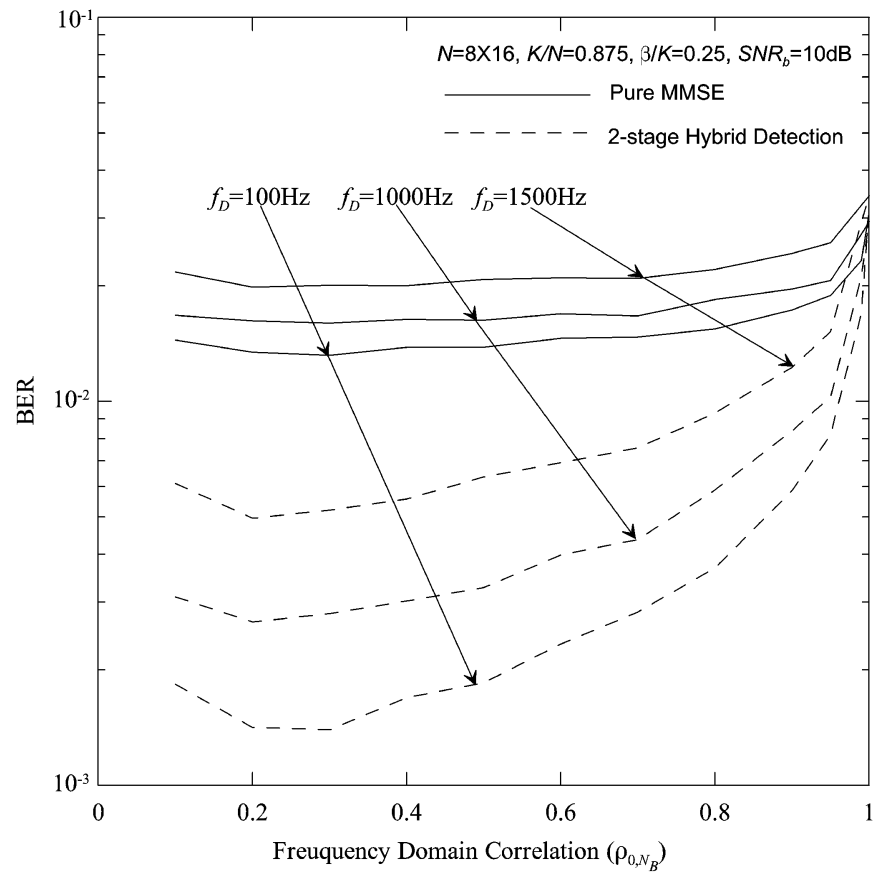

Fig. 8. System performance as a function of subcarrier correlation.

a particular value of $\beta$. Further increasing $\beta$ beyond that value increases BER due to the low transmit power efficiency and more interference caused to data channels by the pilot channel. With given conditions, the optimum $\beta / K$ is located in the range of $[0.2,0.3]$ almost independent of Doppler shift. Similar trends can be observed from pure MMSE, one-stage and three-stage hybrid detection for $f_{D}=1500 \mathrm{~Hz}$. In summary, to provide near-optimum performance, the power ratio $\beta / K$ should be located in the range of $[0.2,0.3]$, irrespective of the Doppler shift and the number of stages.

Fig. 8 illustrates the effect of subcarrier correlation on the BER performance with pure MMSE and two-stage hybrid de-

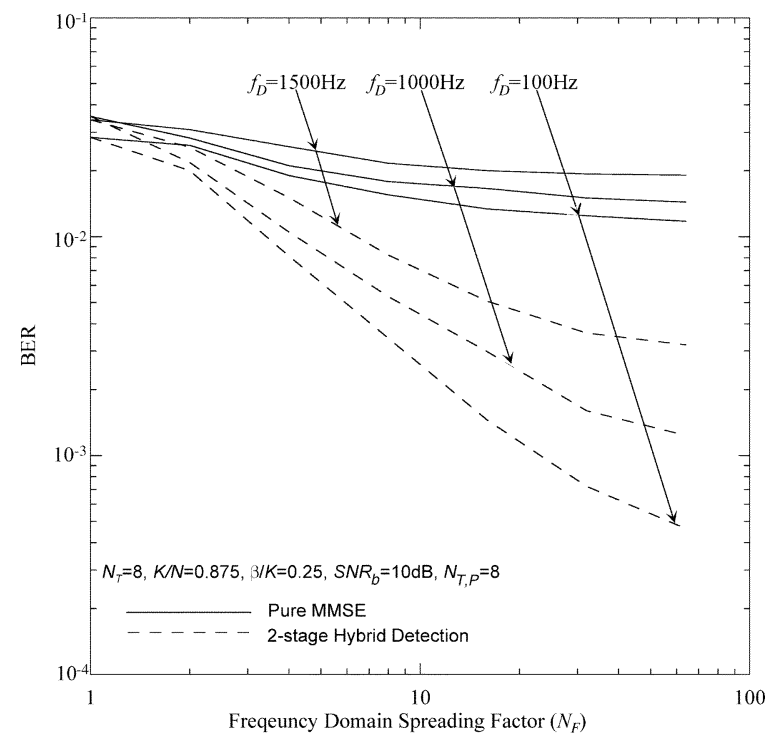

Fig. 9. System performance as a function of $N_{F}$ for a fixed $N_{T}$.

tection. Given a system bandwidth, the number of subcarriers $M$ and spreading scheme $N=N_{T} \times N_{F}$, the correlation coefficient $\rho_{0, N_{B}}$ varies with channel coherence bandwidth $\Delta f_{c}$. Generally, the system performance degrades as $\rho_{0, N_{B}}$ increases due to less frequency diversity gain. It can be seen that for pure MMSE, the degradation is not obvious, whereas for the hybrid detection, the effect of subcarrier correlation is more obvious when $\rho_{0, N_{B}}$ is large and Doppler shift is small. There are also some fluctuations in the BER curves when $\rho_{0, N_{B}}$ is small. This is because with the frequency-domain sliding window size of $2 N_{\text {avg }}+1=5$, the channel estimation quality is related to the subcarrier correlations. The higher the correlation, the more accurate the channel estimation. When the correlation increases from a small value, the system performance is first improved by better channel estimation. However, the frequency diversity gain decreases at the same time. When the loss in frequency diversity is larger than the improvement obtained from better channel estimation, the system performance degrades. Although the performance of the hybrid detection is degraded by a large correlation, there is still significant improvement from hybrid detection as long as $\rho_{0, N_{B}} \leq 0.7$.

The BERs of the system with pure MMSE and two-stage hybrid detection are plotted in Fig. 9 as a function of $N_{F}$ when $N_{T}$ is fixed to eight. It can be seen that with pure MMSE, the system performs poorly with various values of $N_{F}$. However, with the hybrid detection, for both small Doppler $\left(f_{D}=100 \mathrm{~Hz}\right)$ and large Doppler $\left(f_{D}=1000\right.$ and $\left.1500 \mathrm{~Hz}\right)$, the system performance improves significantly when $N_{F}$ increases. This is because although MCI-F increases, the frequency diversity gain also gets larger with increasing $N_{F}$. Furthermore, with MCI cancellation, the gain from diversity overcomes the loss from increased MCI. For $f_{D}=100 \mathrm{~Hz}$, the most significant improvement is observed when $N_{F}$ is increased from 2 to 32 . When $N_{F}$ is further increased from 32, although the BER still decreases, the trend of the reduction is slowed down. This is because the frequency diversity gain becomes saturated when $N_{F}$ is large. For $f_{D}=1000 \mathrm{~Hz}$, similar trends can be observed 
and when $N_{F}$ is increased from 32 to 64 , the performance saturation is more obvious than that for $f_{D}=100 \mathrm{~Hz}$. Finally, for $f_{D}=1500 \mathrm{~Hz}$, it can clearly be seen that the improvement in BER is insignificant when $N_{F}$ is increased from 16. The frequency diversity gain is saturated when $N_{F}=16$ for $f_{D}=1500 \mathrm{~Hz}$. In summary, although the system performance improves when $N_{F}$ increases, the frequency diversity gain saturates when $N_{F}$ is large. The larger the Doppler shift, the smaller $N_{F}$ the performance saturates at.

\section{CONCLUSIONS}

An analytical study is presented on the effect of Doppler shift on the system performance with hybrid MCI cancellation and MMSE detection, when imperfect channel estimation is considered. The following conclusions are drawn:

1) The quality of channel estimation is critical to the performance of the hybrid detection in the presence of Doppler. Using the proposed pilot structure, the window size $N_{T, P}$ in time domain of channel estimation depends on Doppler. When the Doppler shift is small, a large window size of channel estimation is needed, whereas when the Doppler shift is large, a small window size is preferable;

2) MCI-T increases with Doppler shifts. Different data code channels have different BER performance. The data codes adjacent to the pilot code should be assigned with the lowest priority;

3) A two-stage hybrid detection is sufficient for a large range of Doppler shift. Moreover, to provide near optimum performance, the power ratio $\beta / K$ should be located in the range of $[0.2,0.3]$, irrespective of the Doppler shift and the number of stages;

4) Although the system performance improves when $N_{F}$ increases, the frequency diversity gain saturates when $N_{F}$ is large. The larger the Doppler shift, the smaller $N_{F}$ the performance saturates at.

\section{REFERENCES}

[1] S. Abeta, H. Atarashi, M. Sawahashi, and F. Adachi, "Performance of coherent multi-carrier/DS-CDMA and MC-CDMA for broadband packet wireless access," IEICE Trans. Commun., vol. E84-B, no. 3, pp. 406-413, Mar. 2001.

[2] S. Hara and P. Ramjee, "Design and performance of multicarrier CDMA system in frequency-selective Rayleigh fading channels," IEEE Trans. Veh. Technol., vol. 48, no. 5, pp. 1584-1595, Sep. 1999.

[3] E. A. Sourour and M. Nakagawa, "Performance of orthogonal multicarrier CDMA in a multi-path fading channel," IEEE Trans. Commun., vol. 44, no. 3, pp. 356-367, Mar. 1996.

[4] A. Sumasu, T. Nihei, K. Kitagawa, M. Uesugi, and O. Kato, "An OFDM-CDMA system using combination of time and frequency domain spreading," (in Japanese) Tech. Rep. IEICE, RCS2000-3, Apr. 2000.

[5] A. Persson, T. Ottosson, and E. Strom, "Time-frequency localized CDMA for downlink multi-carrier systems," in Proc. IEEE ISSSTA, Sep. 2002, pp. 118-122.

[6] F. Adachi, M. Sawahashi, and H. Suda, "Wideband DS-CDMA for next-generation mobile communications systems," IEEE Commun. Mag., vol. 36, no. 9, pp. 56-69, Sep. 1998.

[7] N. Maeda, H. Atarashi, and M. Sawahashi, "Performance comparison of channel interleaving methods in frequency domain for VSF-OFCDM broadband wireless access in forward link," IEICE Trans. Commun., vol. E86-B, pp. 300-313, Jan. 2003.
[8] H. Atarashi, N. Maeda, S. Abeta, and M. Sawahashi, "Broadband packet wireless access based on VSF-OFCDM and MC/DS-CDMA," in Proc. IEEE PIMRC, Sep. 2002, pp. 992-997.

[9] N. Maeda, Y. Kishiyama, and M. Sawahashi, "Variable spreading factor-OFCDM with two dimensional spreading that prioritizes time domain spreading for forward link broadband wireless access," in Proc. IEEE VTC, Apr. 2003, pp. 127-132.

[10] Y. Kishiyama, N. Maeda, H. Atarashi, and M. Sawahashi, "Investigation of optimum pilot channel structure for VSF-OFCDM broadband wireless access in forward link," in Proc. IEEE VTC, Apr. 2003, pp. 139-144.

[11] H. Atarashi and M. Sawahashi, "Investigation of inter-carrier interference due to Doppler spread in OFCDM broadband packet wireless access," IEICE Trans. Commun., Special Issue Softw. Defined Radio Technol. Appl., vol. 86-B, pp. 291-299, Jan. 2003, 2002SRP-28.

[12] Y. Q. Zhou, J. Wang, and M. Sawahashi, "Downlink transmission of broadband OFCDM systems-Part I: hybrid detection," IEEE Trans. Commun., vol. 53, no. 4, pp. 718-729, Apr. 2005.

[13] K. Witrisal, "OFDM air-interface design for multimedia communications," Ph.D. dissertation, Delft University of Technology, Delft, The Netherlands, Apr. 2002.

[14] W. C. Y. Lee, Mobile Communications Engineering: Theory and Applications, 2nd ed. New York: McGraw-Hill, 1997.

[15] Z. Xing, "Parallel ensemble Monte Carlo for device simulation," in Workshop High-Performance Comput. Activities Singapore, Sep. 1995.

[16] "Technical Specification: Group Radio Access Networks; Deployment Aspects,", 3GPP TR 25.934, V5.1.0, 3GPP, 2002-2006.

[17] R. B. Ertel and J. H. Reed, "Generation of two equal power correlated Rayleigh fading envelopes," IEEE Commun. Lett., vol. 2, no. 10, pp. 276-278, Oct. 1998.

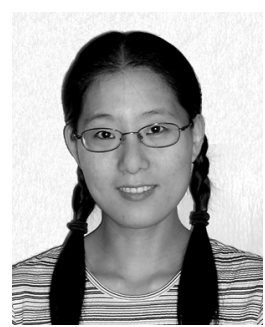

Yiqing Zhou (M'04) was born in Zhejiang, China, in 1975. She received the B.S. degree in communication and information engineering, and the M.S. degree in signal and information processing from the Southeast University, Nanjing, China, in 1997 and 2000 respectively, and the Ph.D. degree in electrical and electronic engineering from the University of Hong Kong, Hong Kong, in 2004.

Since June 2004, she has been with the Department of Electrical and Electronic Engineering, University of Hong Kong, as a Postdoctoral Fellow. Her research interests include coding theory, spread spectrum, OFDM systems, interference cancellation, hybrid ARQ, and other transmission techniques for wireless highspeed data communications.

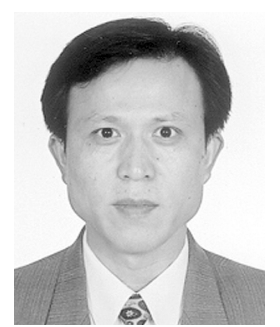

Jiangzhou Wang (M'91-SM'94) received the B.S. and M.S. degrees from Xidian University, Xian, China, in 1983 and 1985, respectively, and the $\mathrm{Ph} . \mathrm{D}$. degree (with Greatest Distinction) from the University of Ghent, Ghent, Belgium, in 1990, all in electrical engineering.

Dr. Wang is currently a Professor and Chair in the Department of Electronics, University of Kent, Kent, U.K. From 1995 to 2005, he was with the University of Hong Kong, where he is still serving as an Honorary Professor. From 1992 to 1995 , he was a Senior System Engineer with Rockwell International Corporation (now Conexant), Newport Beach, CA. From 1990 to 1992, he was a Postdoctoral Fellow with the University of California at San Diego, CA. He held a Visiting Professor position with NTT DoCoMo, Japan. He has published over 140 papers, including more than 40 IEEE TRANSACTIONS/JOURNAL papers in the areas of wireless mobile and spread spectrum communications. He has written/edited two books, Broadband Wireless Communications (Boston, MA: Kluwer, 2001) and Advances in $3 G$ Enhanced Technologies for Wireless Communications (Norwood, MA: Artech House, 2002). The latter book has been translated into Chinese. He holds one U.S. patent in the GSM system.

Dr. Wang is an Editor for IEEE TRANSACTIONS ON COMMUNICATIONS and a Guest Editor for IEEE JOURNAL ON SELECTED AREAS IN COMMUNICATIONS (Wideband CDMA, 2000 and 2001, and Advances in Multicarrier CDMA, 2006). He is listed in Who's Who in the World (New Providence, NJ: Marquis). He was a Technical Chairman of the IEEE Workshop in 3G Mobile Communications in 2000. He has been a Technical Committee Member and Session Chair for a number of international conferences. 


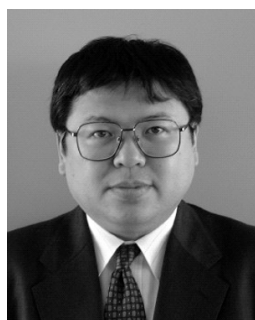

Mamoru Sawahashi (M'88) received the B.S. and M.S. degrees from Tokyo University, Tokyo, Japan, in 1983 and 1985, respectively, and the Dr. Eng. degree from the Nara Institute of Technology, Nara, Japan, in 1998.

In 1985, he joined NTT Electrical Communications Laboratories, and in 1992, he transferred to NTT Mobile Communications Network, Inc. (now NTT DoCoMo, Inc.). Since joining NTT, he has been engaged in the research of modulation/demodulation techniques for mobile radio, and research and development of wireless access technologies for W-CDMA mobile radio and broadband wireless packet access technologies for beyond IMT-2000. He is now the Director of the IP Radio Network Development Department of NTT DoCoMo, Inc.

Dr. Sawahashi is currently serving as an Editor for the IEEE TRANSACTIONS ON WIRELESS COMMUNICATIONS and a Guest Editor for the IEEE JOURNAL ON SELECTED AREAS IN COMMUNICATIONS. 